\title{
KRAS mutant tenosynovial giant cell tumor in a pediatric patient: a case report
}

\author{
Nikhil P. Mankuzhy ${ }^{1,2}$, Bailey Anderson ${ }^{2}$, Amer Heider ${ }^{3}$, Thomas F. Michniacki ${ }^{2}$, Chandan Kumar-Sinha, ${ }^{3,4}$ \\ Rajen Mody ${ }^{2,4,5}$ \\ ${ }^{1}$ Oakland University William Beaumont School of Medicine, Rochester, MI, USA; ${ }^{2}$ Department of Pediatrics, ${ }^{3}$ Department of Pathology, ${ }^{4}$ Center for \\ Translational Pathology, ${ }^{5}$ Rogel Cancer Center, University of Michigan, Ann Arbor, MI, USA \\ Correspondence to: Rajen J. Mody, MD, MS. Professor of Pediatrics, Michigan Medicine, D4205 Medical Professional Building, PO BOX 5718, Ann \\ Arbor, MI, USA. Email: rmody@umich.edu.
}

\begin{abstract}
Tenosynovial giant cell tumors (TSGCT) are a group of rare, benign soft tissue tumors with common histologic and cytogenetic features, with a median age of diagnosis being 47 years. Generally divided into localized and diffuse subtypes, TSGCTs are typically driven by overexpression of macrophage colony stimulating factor receptor-1 (CSF1R). Treatment of TSGCT is tumor resection, followed by radiation therapy in cases of incomplete resection. Even when the tumor is completely removed, recurrence rates can be as high as $30 \%$ in some anatomical locations. Here we report the identification of a previously undescribed KRAS p.G12D activating mutation within a pediatric TSGCT patient, who clinically presented with an enlarging right lower extremity mass pathologically consistent with TSGCT. The patient continues to be in remission three years after complete surgical removal. KRAS mutations are usually found in adult cancers, such as lung and pancreatic, as well as giant cell lesion of the jaw. This case demonstrates the utility of integrative clinical sequencing in identifying lesions with aggressive potential and aiding in complex diagnoses.
\end{abstract}

Keywords: KRAS; tenosynovial giant cell tumor (TSGCT); integrative clinical sequencing; pediatric oncology; case report

Submitted Sep 12, 2019. Accepted for publication Nov 22, 2019.

doi: $10.21037 /$ tp.2019.11.05

View this article at: http://dx.doi.org/10.21037/tp.2019.11.05

\section{Introduction}

Tenosynovial giant cell tumor (TSGCT) is a predominantly benign, neoplastic mass derived from synovium that is also comprised of macrophages, multinucleate giant cells, and other immune cells. It can be classified into localized or diffuse type, formerly referred to as giant cell tumor of the tendon sheath and pigmented villonodular synovitis respectively (1). TSGCT are rare, especially in children, with an incidence rate per million person-years of 2.42 and 1.09 for localized and diffuse types (2). Standard treatment is complete excision with adjuvant radiotherapy used for incomplete surgical resection $(3,4)$. The cytogenetic abnormalities found in the majority of cases are translocations involving chromosome $1 \mathrm{p} 13$ and the colony stimulating factor 1 (CSF1) gene, resulting in overexpression and stimulation of CSF1R causing an accumulation of nonneoplastic cells (5).

Kirsten ras (KRAS) oncogene is one of the most frequently mutated genes in cancer, predominantly found in many adult cancers, including pancreas, colon, and nonsmall cell lung cancer. However, it has not been described in TSGCT (6). In this report, we present the case of the discovery of a previously undescribed activating KRAS p.G12D mutation in a presumed, otherwise benign, diffuse TSGCT in a 10-year-old girl. 


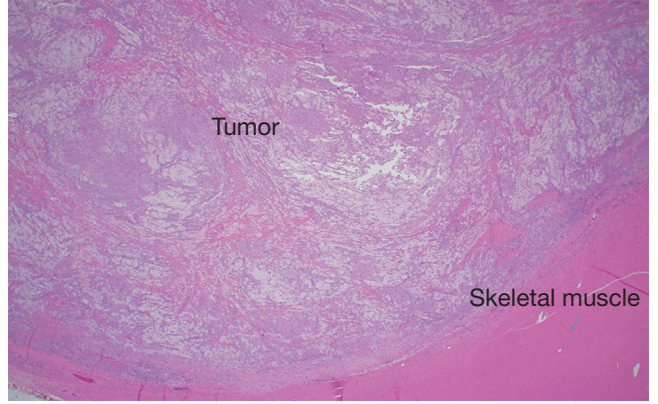

Figure $1 \mathrm{HE}$ stain $2 \times$, showing well circumscribed tumor within skeletal muscle fibers.
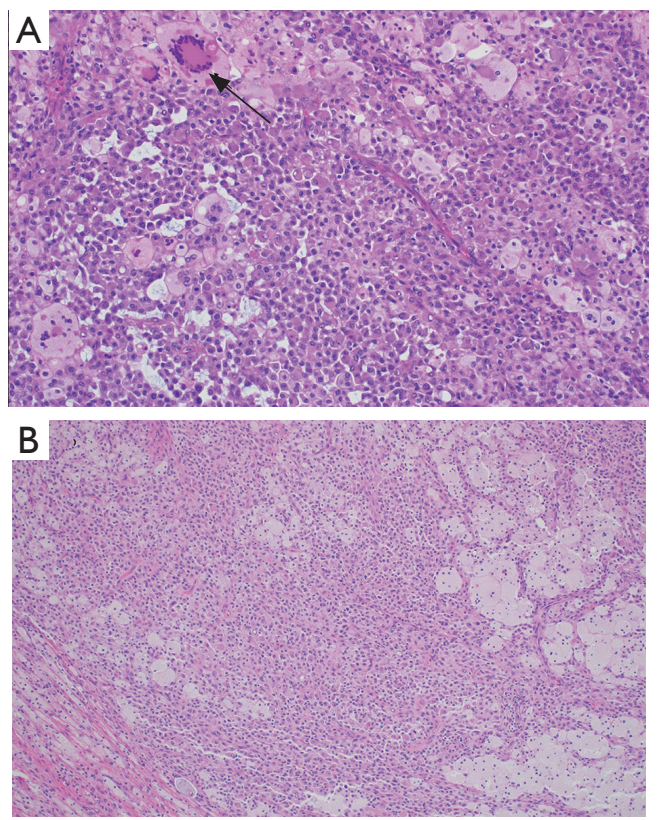

Figure 2 Tumor microscopic photomicrograph. (A) HE stain, 20x magnification, revealing epithelioid mononuclear cells admixed with histiocytes and multinucleated tuton-type giant cells (arrow); (B) $10 \times$ magnification admixed epithelioid neoplastic cells with foamy histiocytes and occasional spindled cells.

\section{Case presentation}

\section{Patient information and clinical findings}

Our patient, an otherwise previously healthy 10 -year-old Caucasian female, initially noted a right posterior thigh mass approximately 1 year prior to her presentation at our institution. The mass was without pain or surrounding erythema and did not limit patient mobility. Clinical history lacked associated fevers, weight loss, night sweats or adenopathy. Physical examination noted a firm, immobile mass within the upper medial posterior right thigh without tenderness to palpation. Laboratory testing showed normal complete blood count with differential, uric acid and comprehensive metabolic panel analyses. The erythrocyte sedimentation rate was normal at $17 \mathrm{~mm}$ with only minimally elevated lactate dehydrogenase and C-reactive protein levels at $325 \mathrm{IU} / \mathrm{L}$ and $0.9 \mathrm{mg} / \mathrm{dL}$, respectively. Given progressive enlargement of the mass, an ultrasound was obtained.

\section{Diagnostic assessment and therapeutic intervention}

Sonography of the mass showed an approximately $4.3 \times 2.8 \times 3.1 \mathrm{~cm}^{3}$ ill-defined predominantly hyperechoic, but heterogeneous soft tissue mass along the right posterior medial thigh musculature with abnormal increased internal vascular flow. The mass was considered nonspecific, but aggressive appearing. Further imaging via right lower extremity magnetic resonance (MR) confirmed a $4.1 \times 4.0 \times 3.0 \mathrm{~cm}^{3}$ soft tissue mass within the semimembranosus muscle. Normal bone marrow signal and no evidence of involvement of the osseous structures were noted. An ultrasound-guided core biopsy of the mass was thus obtained with pathology initially concerning for embryonal rhabdomyosarcoma with the differential diagnosis also including a rhabdomyoma or additional neoplastic processes. Computed tomography (CT) chest/abdomen/ pelvis and positron emission tomography (PET) imaging showed no evidence of metastatic disease or regional spread. The patient underwent a wide excision of the mass with pathology review performed at our institution and two additional outside facilities.

\section{Follow-up and outcomes}

The histologic sections showed well circumscribed epithelioid neoplasm involving skeletal muscle with occasional fibrous strands (Figure 1). The neoplastic cells had epithelioid features with occasional rhabdomyocytelike morphology and admixed xanthomatous and toutontype giant cells (Figures 2,3). Mitotic figures were variable within the tumor. Immunostains showed diffuse positivity for histiocytic marker CD163 (Figure 4). Desmin was also positive and myogenin was negative with rare degenerated cell staining (Figures 5,0). The overall findings were most consistent with tenosynovial giant cell tumor, diffuse type. Due to the uncertainty of the diagnosis from the original 


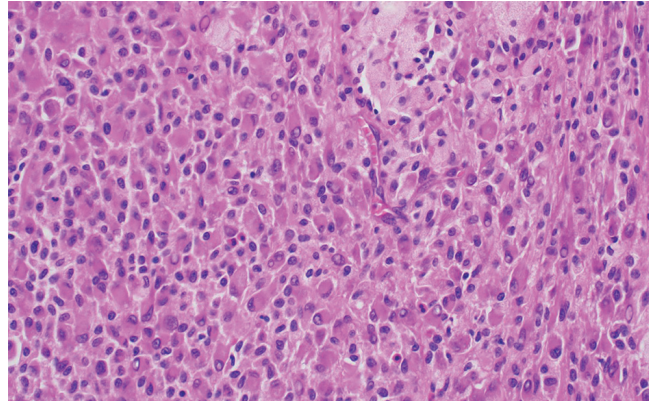

Figure 3 High magnification (40x), HE stain showed epithelioid cells with eccentric nuclei eosinophilic cytoplasm and slight nuclear atypia (rhabdomyocyte-like).

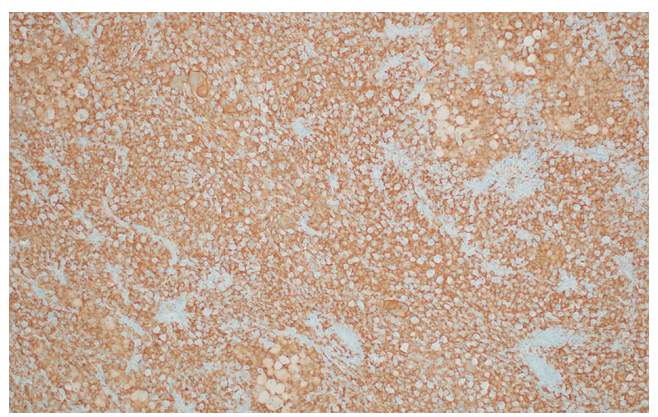

Figure 4 CD163 immunostain, 10× magnification. Diffuse reactivity indicating histiocytic differentiation of this tumor.

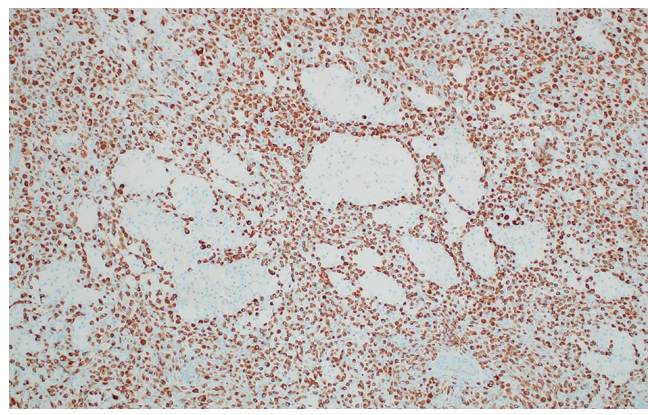

Figure 5 Desmin immunostain, 10x magnification showing unusual diffuse expression that had been reported in subset tenosynovial giant cell tumor, a potential pitfall with rhabdomyosarcoma.

biopsy, patient was consented and enrolled on a prospective, institutional review board approved (HUM00056496), integrative clinical sequencing (ICS) trial (PEDSMIONCOSEQ), in which patient samples undergo paired tumor/normal DNA sequencing, tumor RNA-sequencing, and bioinformatics analyses, details of which have been

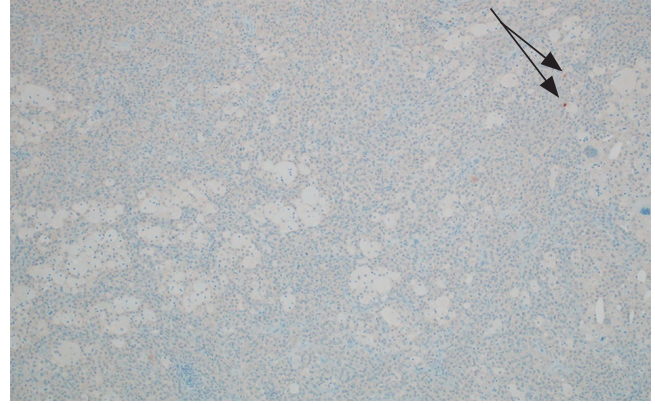

Figure 6 Myogenin immunostain 10x magnification, is negative with rare scattered cell staining (arrows).

previously described (7). The patient's parents provided informed consent and received mandatory pre-enrollment genetic counseling in which no family history of cancer was noted. The results of this analysis are summarized in Table 1 . Two somatic missense mutations were detected, including an unexpected hotspot, activating, mutation of KRAS p.G12D. RNA-seq data from the tumor showed outlier gene expression of CSF1 and CSFR1 (Figure 7), strongly favoring the diagnosis of TSGCT. No pathogenic germline variants in cancer associated genes were identified.

Given complete excision of the mass, no systemic therapy has been administered and the patient has been monitored for local recurrence via physical examinations and MR imaging every 6 months. The patient is now nearly 3 years post-mass excision and doing well with no signs of neoplastic recurrence.

\section{Discussion}

TSGCT is a rare soft tissue tumor, which primarily occurs in the $3^{\text {rd }}$ to $5^{\text {th }}$ decade of life with a median age of 47 years for first treatment (8). Diffuse type can be classified as intraarticular or extraarticular, with a slight predisposition to females in the extraarticular form (9). In both adults and children, they generally present with swelling and pain, and reduced range of motion in intraarticular disease and a soft-tissue mass in extraarticular disease $(2,9)$. While predominantly a benign tumor that can be locally aggressive, recent reports concerning for malignant progression including distant metastasis have been reported. These tumors typically do not contain any atypical features on histological analysis $(10,11)$. Regardless, wide surgical excision followed by consideration for adjuvant externalbeam radiotherapy for improved local control, comprise the 
Table 1 Results of integrative clinical sequencing analysis

\begin{tabular}{lccc}
\hline Methodology/Gene & Protein change & Effect & Variant reads \\
\hline $\begin{array}{l}\text { DNA sequencing } \\
\text { KRAS }\end{array}$ & p.G12D & Missense & $71 / 651(11 \%)$ \\
TRAF7 & p.S561R & Missense & $59 / 536(11 \%)$ \\
RNA sequencing & & & \\
CSF1 & N/A & Outlier expression & 191.3 \\
CSF1R & N/A & Outlier expression & 227.1 \\
ABCA1 & N/A & Outlier expression & 138.6 \\
TGFBR1 & N/A & Outlier expression & 58.7 \\
\hline
\end{tabular}

FPKM, fragments per kilobase of transcript per million mapped reads.

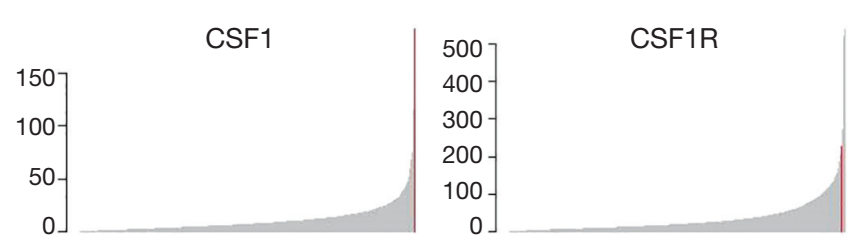

Figure 7 Expression profiles of selected genes from 10-yearold TSGCT patient (in red), in the context of gene expression in our pan-cancer cohort comprising 3,959 samples. TSGCT, tenosynovial giant cell tumor.

primary treatment approach.

While not detected in our patient, some TSGCTs harbor a translocation of chromosome $1 \mathrm{p} 13$ involving the CSF1 gene, commonly with COL6A3 on chromosome 2. This translocation exists in a low percentage of tumor cells, but the majority express the CSF1 receptor (CSF1R). The result of this fusion is increased expression of CSF1, which was observed through transcriptome sequencing in this case. CSF1 mediates proliferation and differentiation of macrophages expressing CSF1R resulting in proliferation and multinucleated giant cell formation. In TSGCT, the neoplastic cells releasing CSF1 are derived from the synovial lining, while the remaining composition of the mass derives from reactive, non-neoplastic CSF1R expressing cells (5). Tyrosine kinase inhibitors or monoclonal antibodies targeting CSF1R, such as imatinib or emactuzumab, represent possible emerging treatment options for patients in-lieu of or in conjunction with surgery for recurrence $(12,13)$.

The KRAS gene is an oncogene that encodes the protein
K-RAS, which is a GTPase that functions as a switch to transmit extracellular signals from receptor tyrosine kinases through the mitogen-activated protein kinase pathway to control numerous cellular mechanisms, such as cell growth and differentiation. Activating KRAS mutations diminish the ability of the protein to regulate signal transduction pathways, leading to cell transformation and increased resistance to therapy $(14,15)$. Activating mutations in RAS account for approximately $25 \%$ of all cancers, with missense mutations at codon 12 being the most common among these (16). KRAS G12D mutations have been found in about $4 \%$ of adult cancers and have shown to be a negative prognostic marker in pancreatic and lung cancers $(17,18)$, and have proven difficult to inhibit clinically due to its structure and intracellular location. Mutations in KRAS are not found frequently in pediatric tumors. A review of St. Jude's Pediatric Cancer Data Portal (accessed July 2019) revealed approximately $4 \%$ of tumor samples harbored mutations in KRAS, with KRAS p.G12D alterations (1.07\%) identified in leukemia $(n=42)$, glioma $(n=4)$, neuroblastoma $(\mathrm{n}=1)$, and Wilms tumor $(\mathrm{n}=1)$ neoplasms.

$K R A S$ mutations, including p.G12D, have been reported frequently in giant-cell lesions of the jaw, which, similar to TSGCT, are typically benign tumors with unpredictable clinical courses (19). In these patients, KRAS mutations are predominantly present in the peripheral form of the lesion, which occurs in the mandible of older individuals. Histopathologically, these lesions generally present as focal distribution of multinuclear giant cells in a spindle cell matrix. Associations between the driving mutations of these lesions and the aggressiveness of the clinical nature of these 
tumors have yet to be investigated.

\section{Conclusions}

Our genetic sequencing findings confirm the diagnosis of TSGCT in a 10-year-old patient, marked by overexpression of $C S F 1 R$, which is a rare diagnosis not typically seen in pediatric patients. The discovery of previously unreported KRASG12D mutation in our case may indicate a greater malignant potential for this tumor, however, since this is a single case it is difficult to draw this conclusion. With some previously reported TSGCT cases noting metastatic capability, an alteration of KRAS could explain a possible molecular mechanism conferring oncogenic potential in a usually benign lesion. This mutation is uncommon in pediatric malignancies, but is most often described in leukemia and has not been previously described in TSGCT. Our case additionally stresses the importance and utility of genetic sequencing in identifying a mutation that can indicate a more aggressive lesion. While our patient has experienced no recurrence or signs of distant metastasis in nearly 3 years, this finding suggests that some patients with TSGCT may benefit from more frequent monitoring. In cases refractory to CSF1R targeting agents, KRAS may provide an additional target for controlling disease with combinations of agents that inhibit the mitogen-activated kinase pathway.

\section{Acknowledgments}

Funding: We acknowledge the support by NIH Clinical Sequencing Exploratory Research (CSER) Award NIH 1UMIH006508.

\section{Footnote}

Conflicts of Interest: R.Mody is a Hyundai Hope on Wheels Scholar. The other authors have no conflicts of interest to declare.

Ethical Statement: The authors are accountable for all aspects of the work in ensuring that questions related to the accuracy or integrity of any part of the work are appropriately investigated and resolved. Written informed consent was obtained from the patient for publication of this case report and any accompanying images. A copy of the written consent is available for review by the Editor-inChief of this journal.

\section{References}

1. de Saint Aubain Somerhausen N, van de Rijn M. Tenosynovial giant cell tumour, localized type Tenosynovial giant cell tumour, diffuse type. In: Fletcher CD, Bridge JA, Hogendoorn P, et al. (eds) World Health Organization classification of tumours of soft tissue and bone. Lyon: IARC, 2013:100-3.

2. Mastboom MJL, Verspoor FGM, Uittenbogaard D, et al. Tenosynovial Giant Cell Tumors in Children: A Similar Entity Compared With Adults. Clin Orthop Relat Res 2018;476:1803-12.

3. Aurégan JC, Bohu Y, Lefevre N, et al. Primary arthroscopic synovectomy for pigmented villonodular synovitis of the knee: Recurrence rate and functional outcomes after a mean follow-up of seven years. Orthop Traumatol Surg Res 2013;99:937-43.

4. Duan Y, Qian J, Chen K, et al. Necessity of adjuvant postoperative radiotherapy for diffuse pigmented villonodular synovitis of the knee: A case report and literature review. Medicine (Baltimore) 2018;97:e9637.

5. West RB, Rubin BP, Miller MA, et al. A landscape effect in tenosynovial giant-cell tumor from activation of CSF1 expression by a translocation in a minority of tumor cells. Proc Natl Acad Sci U S A 2006;103:690-5.

6. Kawada K, Toda K, Sakai Y. Targeting metabolic reprogramming in KRAS-driven cancers. Int J Clin Oncol 2017;22:651-9.

7. Mody RJ, Wu YM, Lonigro RJ, et al. Integrative Clinical Sequencing in the Management of Refractory or Relapsed Cancer in Youth. JAMA 2015;314:913-25.

8. Mastboom MJL, Verspoor FGM, Verschoor AJ, et al. Higher incidence rates than previously known in tenosynovial giant cell tumors. Acta Orthop 2017;88:688-94.

9. Murphey MD, Rhee JH, Lewis RB, et al. Pigmented Villonodular Synovitis: Radiologic Pathologic Correlation. Radiographics 2008;28:1493-518.

10. Bertoni F, Unni KK, Beabout JW, et al. Malignant Giant Cell Tumor of the Tendon Sheaths and Joints (Malignant Pigmented Villonodular Synovitis). Am J Surg Pathol 1997;21:153-63.

11. Somerhausen NS, Fletcher CD. Diffuse-Type Giant Cell Tumor. Am J Surg Pathol 2000;24:479-92.

12. Cassier PA, Gelderblom H, Stacchiotti S, et al. Efficacy of imatinib mesylate for the treatment of locally advanced and/or metastatic tenosynovial giant cell tumor/pigmented villonodular synovitis. Cancer 2012;118:1649-55. 
13. Cassier PA, Italiano A, Gomez-Roca CA, et al. CSF1R inhibition with emactuzumab in locally advanced diffusetype tenosynovial giant cell tumours of the soft tissue: a dose-escalation and dose-expansion phase 1 study. Lancet Oncol 2015;16:949-56.

14. Jancík S, Drábek J, Radzioch D, et al. Clinical relevance of KRAS in human cancers. J Biomed Biotechnol 2010;2010:150960.

15. Murray S, Dahabreh IJ, Linardou H, et al. Somatic Mutations of the Tyrosine Kinase Domain of Epidermal Growth Factor Receptor and Tyrosine Kinase Inhibitor Response to TKIs in Non small Cell Lung Cancer: An Analytical Database. J Thorac Oncol 2008;3:832-9.

16. Pylayeva-Gupta Y, Grabocka E, Bar-Sagi D. RAS

Cite this article as: Mankuzhy NP, Anderson B, Heider A, Michniacki TF, Kumar-Sinha C, Mody R. KRAS mutant tenosynovial giant cell tumor in a pediatric patient: a case report. Transl Pediatr 2019;8(5):449-454. doi: 10.21037/ tp.2019.11.05 oncogenes: weaving a tumorigenic web. Nat Rev Cancer 2011;11:761-74.

17. Bournet B, Muscari F, Buscail C, et al. KRAS G12D Mutation Subtype Is A Prognostic Factor for Advanced Pancreatic Adenocarcinoma. Clin Transl Gastroenterol 2016;7:e157.

18. Slebos RJC, Kibbelaar RE, Dalesio O, et al. K-ras Oncogene Activation as a Prognostic Marker in Adenocarcinoma of the Lung. N Engl J Med 1990;323:561-5.

19. Gomes CC, Gayden T, Bajic A, et al. TRPV4 and KRAS and FGFR1 gain-of-function mutations drive giant cell lesions of the jaw. Nat Commun 2018;9:4572. 